\title{
Impact of a Newly Developed Short Double-Balloon Enteroscope on Stent Placement in Patients with Surgically Altered Anatomies
}

\author{
Koichiro Tsutsumi, Hironari Kato, and Hiroyuki Okada
}

Department of Gastroenterology and Hepatology, Okayama University Graduate School of Medicine, Dentistry, and Pharmaceutical Sciences, Okayama, Japan

A newly developed short double-balloon enteroscope with a working channel enlarged to a diameter of $3.2 \mathrm{~mm}$ is a novel innovation in stent placement for patients with surgically altered anatomies. Herein, we report three patients in whom this new scope contributed to an efficient technique and ideal treatment. In the first case, the double guidewire technique was efficient and effective for multiple stent placements. In the second case, covered self-expandable metal stent (SEMS) placement, which is the standard treatment for malignant biliary obstruction, could be performed in a technologically sound and safe manner. In the third case, SEMS placement was performed as palliative treatment for malignant afferent-loop obstruction; this procedure could be performed soundly and safely using the through-the-scope technique. The wider working channel of this new scope also facilitates a smoother accessory insertion and high suction performance, which reduces procedure time and stress on endoscopists. Furthermore, this new scope, which has advanced force transmission, adaptive bending, and a smaller turning radius, is expected to be highly successful in both diagnosis and therapy for various digestive diseases in patients with surgically altered anatomies. (Gut Liver 2017;11:306-311)

Key Words: Double-balloon enteroscopy; Cholangiopancreatography, endoscopic retrograde; Stents; Gastric outlet obstruction; Technology

\section{INTRODUCTION}

The short double-balloon enteroscope (DBE) is considered a useful device for endoscopic retrograde cholangiopancreatography (ERCP) in patients with surgically altered anatomies. ${ }^{1}$ One reason for this is that the short length of the scope allows many conventional ERCP accessories to be used, unlike the long DBE. However, the narrow working channel of the scope, with a 2.8$\mathrm{mm}$ diameter, limits the procedures and treatments that can be performed.

On the other hand, a useful overtube-assisted technique with the long single-balloon enteroscope replaced by a conventional forward-viewing upper endoscope, which enables the use of conventional accessories after reaching the target site, was reported. ${ }^{2}$ However, the scope exchange can be difficult, especially if the overtube collapses due to an acute angulated site, ${ }^{3}$ and the diameter of the working channel of currently available upper scopes is only $2.8-\mathrm{mm}$.

A newly developed short DBE (EI-580BT; Fujifilm, Tokyo, Japan), whose working channel is enlarged to $3.2 \mathrm{~mm}$ in diameter (Figs 1 and 2), represents a novel innovation for patients with surgically altered anatomies. Herein, we report three patients in whom this new scope contributed to efficient technique

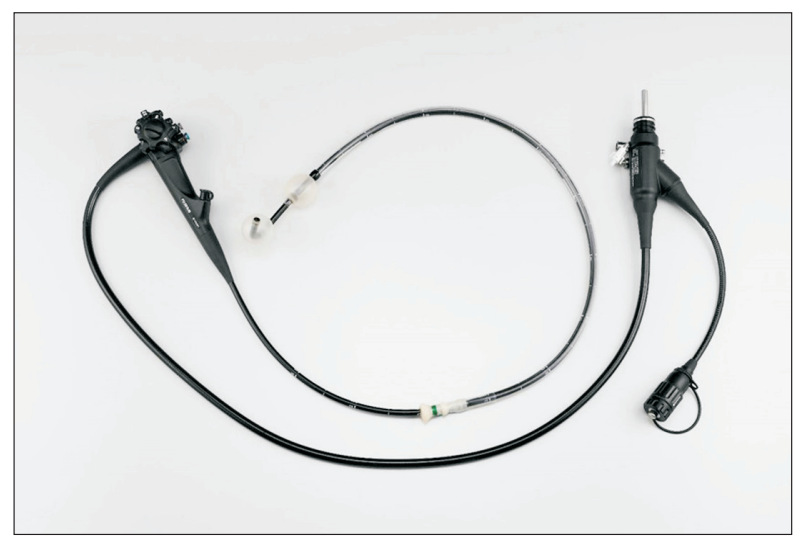

Fig. 1. Newly developed short double-balloon enteroscope.

\footnotetext{
Correspondence to: Koichiro Tsutsumi

Department of Gastroenterology and Hepatology, Okayama University Graduate School of Medicine, Dentistry, and Pharmaceutical Sciences, 2-5-1 Shikata-cho, Kita-ku, Okayama 700-8558, Japan

Tel: +81-86-235-7219, Fax: +81-86-225-5991, E-mail: tsutsumi@cc.okayama-u.ac.jp

Received on September 5, 2016. Revised on October 21, 2016. Accepted on October 21, 2016. Published online February 17, 2017 pISSN 1976-2283 eISSN 2005-1212 https://doi.org/10.5009/gnl16441

@ This is an Open Access article distributed under the terms of the Creative Commons Attribution Non-Commercial License (http://creativecommons.org/licenses/by-nc/4.0) which permits unrestricted non-commercial use, distribution, and reproduction in any medium, provided the original work is properly cited.
} 


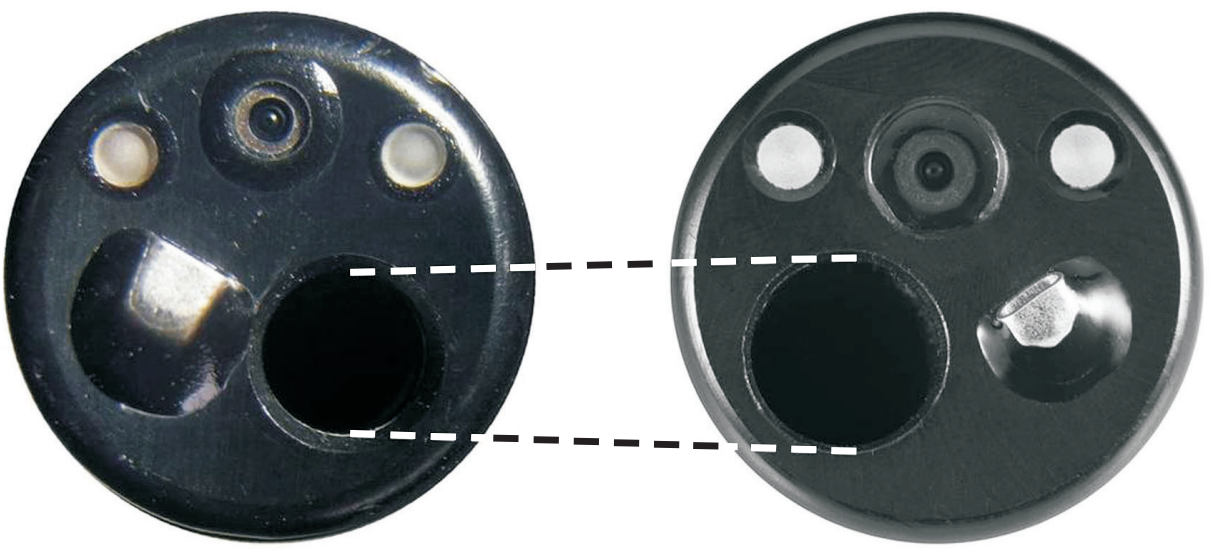

Fig. 2. Distal end layout of the short double-balloon enteroscope. (A) Conventional type (EC-450BI5; Fujifilm); (B) new type (EI-580BT; Fujifilm).
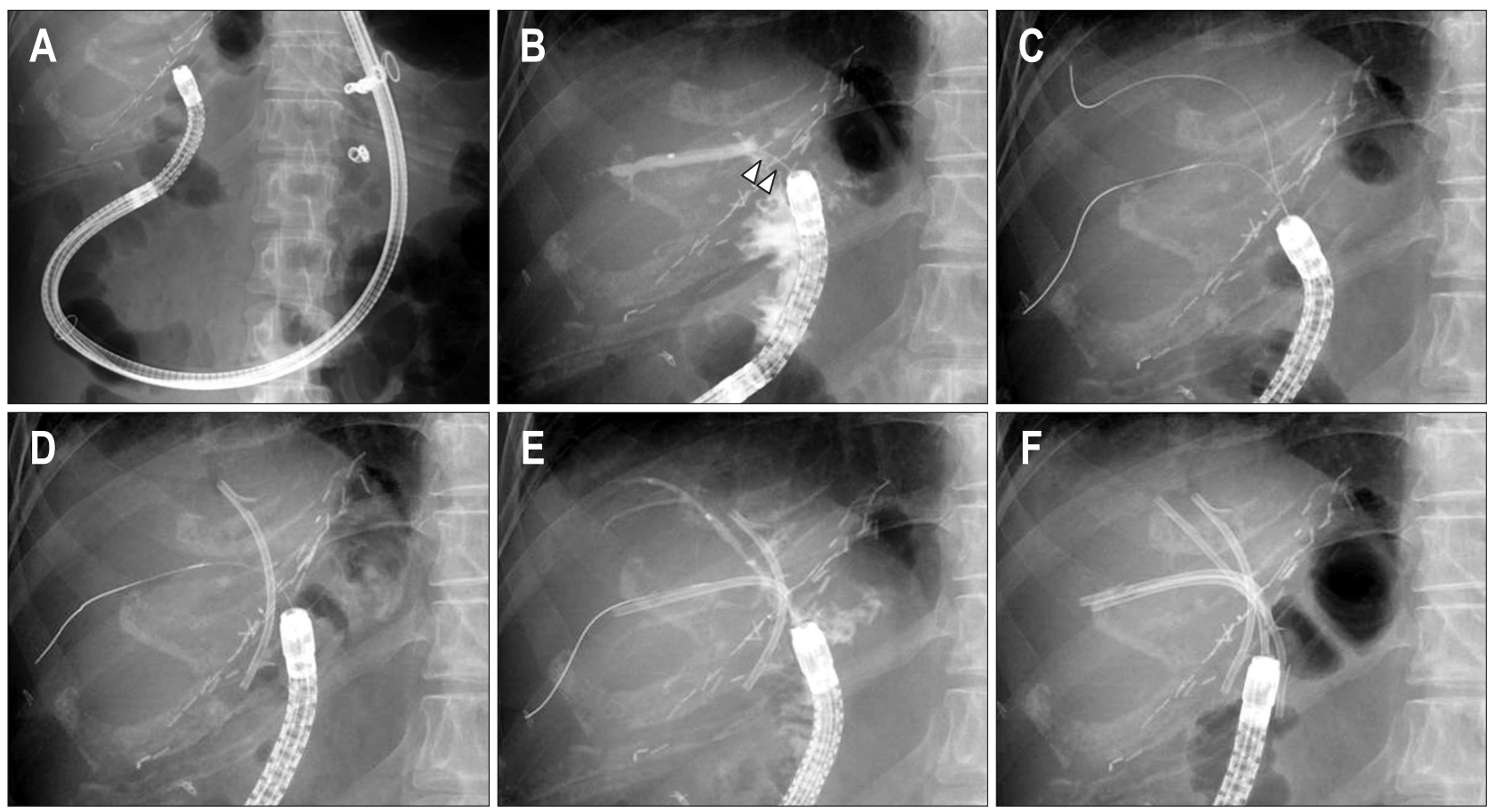

Fig. 3. Multiple plastic stent placements for benign biliary strictures after hepaticojejunostomy with a double guidewire technique using the newly developed short double-balloon enteroscope. (A) The scope reached the bilioenteric anastomotic site. (B) Cholangiogram showed a biliary obstruction (arrowheads, biliary stricture). (C) 0.035-inch and 0.025-inch guidewires were inserted in B7 and B6, respectively. (D) While maintaining the 0.025-inch guidewire in B6, a 7-F plastic stent was smoothly advanced and placed into B7 using a 0.035-inch guidewire. (E) Similarly, after a second 7-F plastic stent was placed into B6 using the reinserted guidewire, the 0.035-inch guidewire was reinserted in B8, and then, a third 7-F plastic stent was placed into B8. (F) Finally, a fourth 7-F plastic stent was easily placed into B6 using the 0.025-inch guidewire that was initially inserted.

and ideal treatment for stent placement.

\section{CASE REPORTS}

\section{Case 1}

A 37-year-old woman who had undergone living donor liver transplantation with right lobe graft for congenital biliary atresia was admitted for cholangitis due to benign recurrent biliary obstruction. She had a history of hepaticojejunostomy as a re- construction method. Therefore, ERCP was attempted for biliary drainage using the newly developed short DBE. Scope insertion to the bilioenteric anastomosis was successfully achieved (Fig. $3 \mathrm{~A}$ ), and cholangiogram showed benign strictures of the bile duct (B6, B7, and B8) and bilioenteric anastomotic stricture (Fig. 3B). We planned to place as many plastic stents as possible in order to achieve sufficient dilation of these strictures. First, 0.035-inch (RevoWave; Piolax, Kanagawa, Japan) and 0.025-inch (Visiglide2; Olympus, Tokyo, Japan) guidewires were 


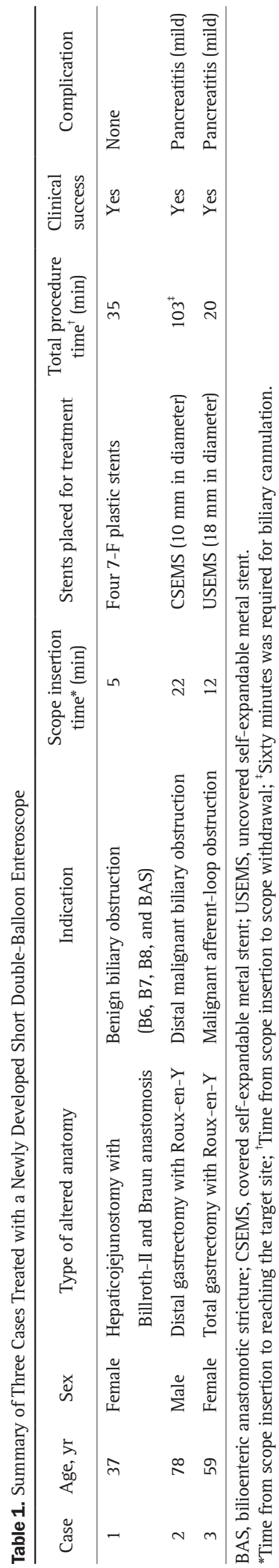

inserted in B7 and B6, respectively (Fig. 3C). While maintaining a 0.025-inch guidewire in B6, a 7-F plastic stent (Through Pass; Gadelius, Tokyo, Japan) was smoothly advanced using a 0.035inch guidewire, and was placed into B7 (Fig. 3D). Secondly, the 0.035-inch guidewire was reinserted in B6, and a second 7-F plastic stent (Through Pass; Gadelius) was placed into B6 using the guidewire, still maintaining the 0.025-inch guidewire in place. Similarly, the 0.035 -inch guidewire was reinserted in B8, and a third 7-F plastic stent (Through Pass; Gadelius) was placed into B8 (Fig. 3E). Finally, a fourth 7-F plastic stent (Through Pass; Gadelius) was successfully placed into B6 using the 0.025-inch guidewire that was initially inserted (Fig. 3F). No procedure-related adverse events occurred. Three months later, these four plastic stents were exchanged safely and accurately by the same methods due to persistent biliary strictures, which were improved but still present (Table 1).

\section{Case 2}

A 78-year-old man who had received chemotherapy for postoperative recurrence of gastric cancer in the intra-abdominal lymph nodes and lung after distal gastrectomy with Roux-en-Y reconstruction was admitted for jaundice. Computed tomography imaging revealed distal malignant biliary obstruction (MBO) due to enlarged lymph nodes. Therefore, ERCP was attempted for biliary drainage using the newly developed short DBE. After insertion of the scope to the ampulla of Vater (Fig. 4A), biliary cannulation was successfully performed; a cholangiogram showed a 15-mm long distal biliary obstruction (Fig. 4B). Following endoscopic sphincterotomy using a sphincterotome (RotaCut; Medi-Globe GmbH, Achenmühle, Germany), a partially covered self-expandable metal stent (CSEMS) with a delivery system of 8.5-F diameter and 194-cm working length (Wallflex biliary RX stent; Boston Scientific, Natick, MA, USA; 10 mm×60 $\mathrm{mm}$ ) was successfully deployed (Fig. 4C and D). Postprocedure, mild pancreatitis occurred, but improved immediately with conservative therapy. After resolution of jaundice, chemotherapy was resumed, and no recurrent biliary obstruction occurred for 13 months (Table 1).

\section{Case 3}

A 59-year-old woman who had undergone total gastrectomy with Roux-en-Y due to gastric cancer was admitted for abdominal pain due to cancer recurrence. Computed tomography imaging revealed malignant afferent-loop obstruction, involving the third portion of the duodenum. Therefore, duodenal stent placement was attempted as palliative therapy using the newly developed short DBE. After reaching the lesion (Fig. 5A), small-bowel enema showed stenosis of approximately $40-\mathrm{mm}$ in length (Fig. 5B). Then, an uncovered self-expandable metal stent (USEMS) (Niti-S D pyloric/duodenal uncovered stent; Taewoong Medical, Gimpo, Korea; 18-mm in diameter, 6-cm in length) with a delivery system of 9-F diameter and 220-cm working length was 

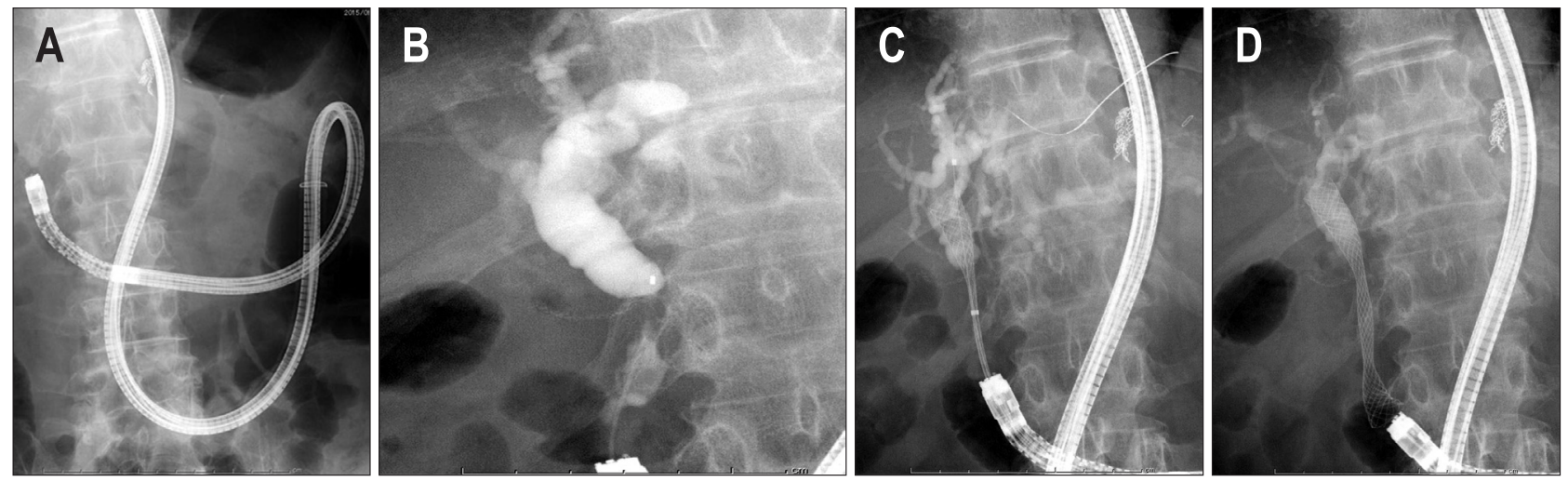

Fig. 4. Transpapillary placement of a partially covered self-expandable metal stent (CSEMS) for distal malignant biliary obstruction (MBO) using the newly developed short double-balloon enteroscope. (A) The scope reached the ampulla of Vater. (B) Cholangiogram showed an approximately 15-mm-long MBO. (C) After smooth advancement of the delivery system into the bile duct, placement of the CSEMS was initiated. (D) Successful transpapillary placement of the partially CSEMS for distal MBO was achieved.
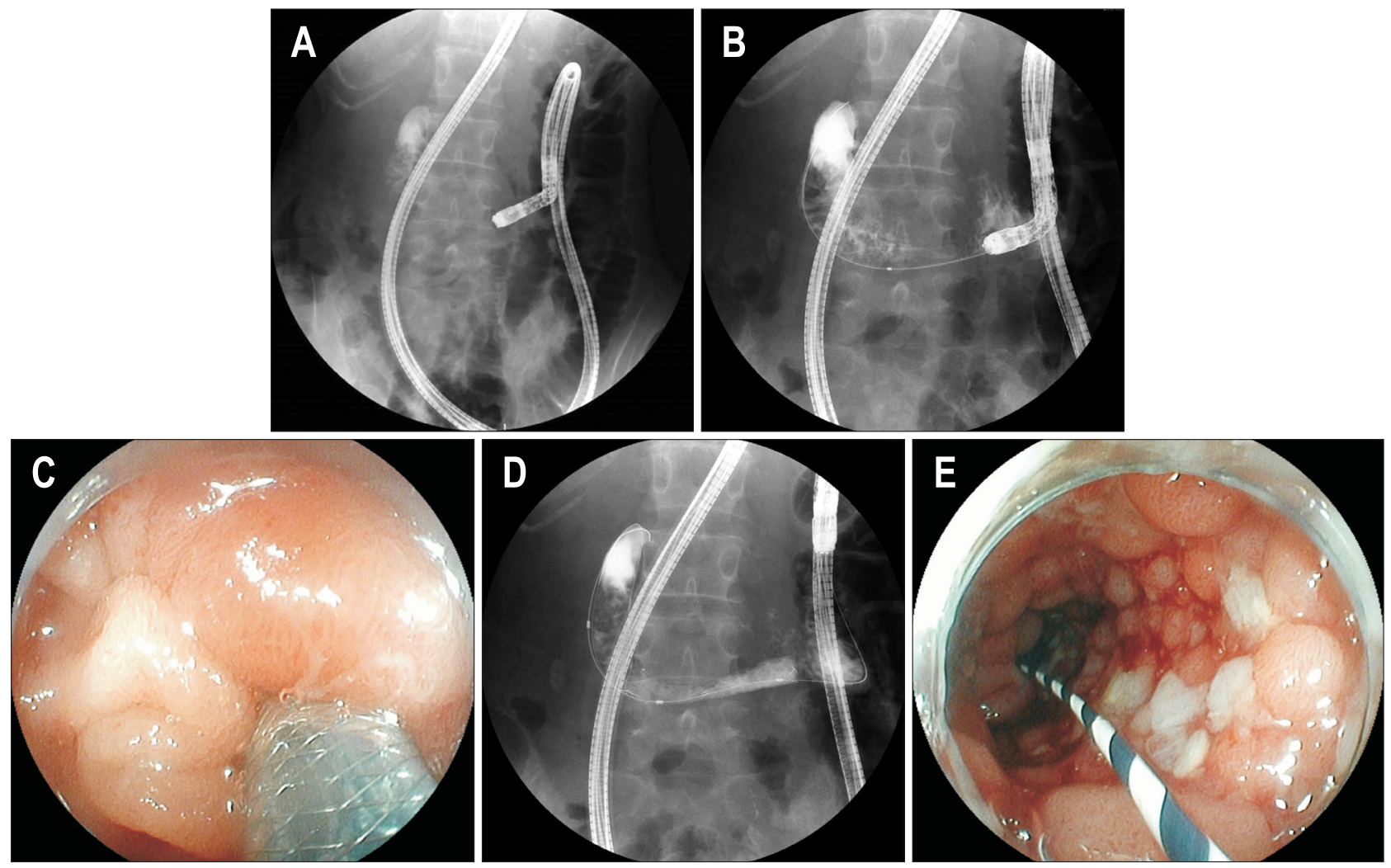

Fig. 5. Metal stent placement for afferent-loop obstruction using the newly developed short double-balloon enteroscope. (A) The scope reached the obstruction site. (B) Small-bowel enema showed an afferent-loop obstruction approximately $40 \mathrm{~mm}$ in length. (C) Endoscopic view showing malignant intestinal stricture, through which the metal stent with a delivery system was advanced. (D) An uncovered self-expandable metal stent was placed correctly and safely using through-the-scope methods. (E) Endoscopic view 1 month after stent placement.

smoothly advanced via the through-the-scope technique, and placed correctly and safely under endoscopic view and fluoroscopic guidance using the new scope (Fig. 5C-E). Postprocedure, mild pancreatitis occurred, but improved immediately with conservative therapy. Thereafter, her symptom also improved (Table 1).

\section{DISCUSSION}

This newly developed short DBE with an enlarged working channel represents technological innovation in endoscopic stent placement for patients with surgically altered anatomies; the double guidewire technique was efficient and effective for 
multiple stent placement. CSEMS placement, which represents standard treatment for MBO, and USEMS placement, as palliative treatment for malignant afferent-loop obstruction, were both performed in a technologically sound and safe manner.

First, we demonstrated multiple 7-F plastic stent placements with the double guidewire technique using standard guidewires, such as 0.035-inch and 0.025-inch guidewires, employing the newly developed short DBE. The conventional short DBE with a small, 2.8-mm working channel does not allow placement of a 7-F stent while also maintaining a second standard guidewire in place. Therefore, we would need to perform biliary stenting one by one: a repeated process consisting of targeting the bile duct using a standard guidewire and placing a plastic stent within the duct. However, this impractical procedure carries a potential risk with the increasing number of stents placed: a greater number of stents would make it more difficult to identify and target the bile duct using the guidewire. Previously, we reported that a 0.018-inch guidewire was useful in the double guidewire technique as a landmark guidewire when performing partial stentin-stent placement of metallic stents using conventional short DBE. ${ }^{4}$ However, a 0.018-inch guidewire is inadequate for biliary stenting, because of its reduced stiffness. The ability to maintain a 0.025-inch guidewire is more useful and reasonable for definitive and efficient biliary stent placement.

In the second case, we describe a patient with postoperative distal MBO due to lymph node metastases, in whom the newly developed short DBE contributed to transpapillary CSEMS placement, by through-the-scope methods. CSEMS is useful for the treatment of distal MBO, because of its long-term patency compared with USEMS. ${ }^{5,6}$ However, although successful scope insertion and cholangiogram were achieved using a conventional short DBE, a CSEMS that can be placed using the short DBE with a small working channel was unavailable; therefore, an USEMS or plastic stent must be selected for distal MBO. The availability of not only USEMS but also CSEMS is critically important from a clinical perspective, because it allows postoperative patients with distal MBO to receive the standard treatment, similar to that used in patients with normal anatomies.

Thirdly, we report a postoperative patient with malignant afferent-loop obstruction, in whom the new scope contributed to the placement of the USEMS, designed as a duodenal stent, by through-the-scope methods. Conventional DBE could never allow gastrointestinal metal stent placement for malignant gastrointestinal obstruction such as an afferent-loop obstruction, because of the narrow working channel of the scope. Therefore, one common practice is to insert the conventional DBE to the stricture and subsequently remove it, leaving the overtube; the self-expandable metal stent is then placed through the overtube under fluoroscopic guidance. ${ }^{7,8}$ This technique is useful, but stent placement using the new scope, and the through-thescope method, as in our case, is more ideal for achieving correct and safe stent placement. $^{9}$
The wider working channel of this new scope also facilitates smoother accessory insertion and high suction performance, which can reduce procedure time and stress on endoscopists. In addition, this new scope allows for improved and easier scope insertion, by producing gradual stiffness with advanced force transmission for better stability and adaptive bending. Additionally, the smaller turning radius allows for improved viewing of the target site and a better environment for treatment. This scope is expected to be useful in improving the ability to obtain successful diagnosis and therapy for various digestive diseases in patients with surgically altered anatomies.

This newly developed short DBE brings about technological innovation in stent placement, and therefore standard, efficient, and safe treatment can be performed even in patients with surgically altered anatomies.

\section{CONFLICTS OF INTEREST}

No potential conflict of interest relevant to this article was reported.

\section{REFERENCES}

1. Sakakihara I, Kato H, Muro S, et al. Double-balloon enteroscopy for choledochojejunal anastomotic stenosis after hepato-biliarypancreatic operation. Dig Endosc 2015;27:146-154.

2. Itoi T, Ishii K, Sofuni A, et al. Single-balloon enteroscopy-assisted ERCP in patients with Billroth II gastrectomy or Roux-en-Y anastomosis (with video). Am J Gastroenterol 2010;105:93-99.

3. Soh JS, Yang DH, Lee SS, et al. Single balloon enteroscopy-assisted endoscopic retrograde cholangiopancreatography in patients who underwent a gastrectomy with Roux-en-Y anastomosis: six cases from a single center. Clin Endosc 2015;48:452-457.

4. Tsutsumi K, Kato H, Okada H, Yamamoto K. A novel technique for partial stent-in-stent placement of three metal biliary stents using a short double-balloon enteroscope. Endoscopy 2014;46 Suppl 1:E417-E418.

5. Saleem A, Leggett CL, Murad MH, Baron TH. Meta-analysis of randomized trials comparing the patency of covered and uncovered self-expandable metal stents for palliation of distal malignant bile duct obstruction. Gastrointest Endosc 2011;74:321-327.e3.

6. Kawakubo K, Isayama H, Nakai Y, et al. Efficacy and safety of covered self-expandable metal stents for management of distal malignant biliary obstruction due to lymph node metastases. Surg Endosc 2011;25:3094-3100.

7. Fujii M, Ishiyama S, Saito H, et al. Metallic stent insertion with double-balloon endoscopy for malignant afferent loop obstruction. World J Gastrointest Endosc 2015;7:665-669.

8. Sasaki T, Isayama H, Kogure H, et al. Double-balloon enteroscope-assisted enteral stent placement for malignant afferentloop obstruction after Roux-en-Y reconstruction. Endoscopy 2014;46(Suppl 1):E541-E542. 
9. Shimatani M, Takaoka M, Tokuhara M, et al. Through-the-scope self-expanding metal stent placement using newly developed short double-balloon endoscope for the effective management of malignant afferent-loop obstruction. Endoscopy 2016;48(Suppl 1):E6-E7. 